\title{
Preoperative N-terminal pro-B-type natriuretic peptide for prediction of acute kidney injury after non-cardiac surgery: A retrospective cohort study
}

\section{Bingcheng Zhao}

Southern Medical University Nanfang Hospital

\section{Pei-Pei Zhuang}

Southern Medical University Nanfang Hospital

Shao-Hui Lei

Southern Medical University Nanfang Hospital

Shi-Da Qiu

Southern Medical University Nanfang Hospital

Xiao Yang

Southern Medical University Nanfang Hospital

Cai Li

Southern Medical University Nanfang Hospital

Wei-Feng Liu

Southern Medical University Nanfang Hospital

Ke-xuan Liu ( $D$ liukexuan705@163.com )

Southern Medical University Nanfang Hospital https://orcid.org/0000-0003-0221-366X

\section{Research}

Keywords: acute kidney injury, natriuretic peptide, non-cardiac surgery, risk prediction

Posted Date: April 24th, 2020

DOI: https://doi.org/10.21203/rs.2.23625/v2

License: (1) (1) This work is licensed under a Creative Commons Attribution 4.0 International License. Read Full License 
The authors have withdrawn this preprint from Research Square 